\title{
SOME INEQUALITIES FOR THE MULTIPLICATOR OF A FINITE GROUP
}

\author{
MICHAEL R. JONES
}

\begin{abstract}
The paper is devoted to the derivation of certain upper and lower bounds for the multiplicator of a finite group. The lower bounds enable us to give a sufficient condition for a finite group to have nontrivial multiplicator.
\end{abstract}

1. Introduction. In this note, some inequalities for the multiplicator, $M(G)$, of a finite group $G$ are given. The main results can also be obtained by applying the spectral sequence due to R. C. Lyndon [5]. However, I feel it is worth while proving them directly using a result due to I. Schur [7] embodied in 1.1 below.

The definition of the multiplicator of a finite group may be found in [3, Kap V, §23], as may the basic facts about multiplicators.

Notation. The notation used is as follows: the exponent of a finite group $X$ is denoted by $e(X)$; if $x$ and $y$ are two elements of some group then $x^{-1} y^{-1} x y$ is denoted by $[x, y]$ and $y^{-1} x y$ is denoted by $x^{y}$; if $X$ and $Y$ are two subgroups of a group $G$ then $[X, Y]$ is the subgroup of $G$ generated by all $[x, y]$ with $x$ in $X$ and $y$ in $Y$; finally, the lower central series of $a$ group $G$ is denoted by $G=\gamma_{1}(G) \geqq \gamma_{2}(G)=G^{\prime} \geqq \gamma_{3}(G) \geqq \cdots$, where for $j \geqq 1, \gamma_{j+1}(G)=\left[\gamma_{j}(G), G\right]$.

All other notation, where not explained, will be standard.

1.1. Let $G$ be a finite group and $G=F / R$ a presentation for $G$ as a factorgroup of the free group $F$. Then

$$
M(G) \cong\left(F^{\prime} \cap R\right) /[F, R] .
$$

2. Some lemmas. The results of this secticn are two lemmas. The proof of the first lemma is much the same as that of Lemma 2.1 of [4] and so will be omitted. The lemmas are used to give the results of $\S \S 3$ and 4. In applying Lemma 2.1 and its corollary, the reader should recall that, for two finite groups $A$ and $B$,

$$
A \otimes B \cong\left(A / A^{\prime}\right) \otimes\left(B / B^{\prime}\right) .
$$

Received by the editors August 1, 1972 and, in revised form, December 4, 1972. AMS (MOS) subject classifications (1970). Primary 20C25; Secondary $20 \mathrm{D} 15$. Key words and phrases. Multiplicator, p-group, central subgroup.

(c) American Mathematical Society 1973 
Lemma 2.1. Let $G$ be $a$ finite group and $B$ a normal subgroup. Set $A=G / B$. Let $G=F / R$ be a presentation for $G$ as a factor-group of the free group $F$ and suppose $B=S / R$ so that $A \cong F / S$. Then $[F, S] /[F, R][F, S, F] S^{\prime}$ is isomorphic with a factor-group of $A \otimes B$.

COROLlARY 2.2 (SEE [4]). Further to the notation and assumptions of Lemma 2.1 , let $B$ be a central subgroup of $G$. Then $[F, S] /[F, R] S^{\prime}$ is an epimorphic image of $A \otimes B$.

LEMMA 2.3. Let $G$ be a finite group with a normal subgroup $K$ and set $H=G / K$. Then there exists a finite group $L$ with a normal subgroup $M$ such that

(i) $G^{\prime} \cap K \cong L / M$,

(ii) $M \cong M(G)$,

(iii) $M(H)$ is an epimorphic image of $L$.

Proof. Let $G=F / R$ be a presentation for $G$ as a factor-group of the free group $F$ and suppose $K=T / R$ so that $H \cong F / T$. Then

$$
\begin{aligned}
G^{\prime} \cap K & =\left(F^{\prime} R \cap T\right) / R=\left(F^{\prime} \cap T\right) R / R \\
& \cong\left(F^{\prime} \cap T\right) /\left(F^{\prime} \cap R\right) \cong\left(\left(F^{\prime} \cap T\right) /[F, R]\right) /\left(\left(F^{\prime} \cap R\right) /[F, R]\right)
\end{aligned}
$$

so that (i) and (ii) follow using 1.1.

Next,

$$
M(H) \cong\left(F^{\prime} \cap T\right) /[F, T] \cong\left(\left(F^{\prime} \cap T\right) /[F, R]\right) /([F, T] /[F, R])
$$

so that (iii) holds.

Note that the kernel of the epimorphism described in Lemma 2.3(iii) is $[F, T] /[F, R]$. We shall use this fact in $\S 4$.

3. Lower bounds. The main result of this section is:

THEOREM 3.1. Let $G$ be a finite group and $K$ any normal subgroup. Set $H=G / K$. Then

(i) $|M(H)|$ divides $|M(G)|\left|G^{\prime} \cap K\right|$,

(ii) $e(M(H))$ divides $e(M(G)) e\left(G^{\prime} \cap K\right)$,

(iii) $d(M(H)) \leqq d(M(G))+d\left(G^{\prime} \cap K\right)$.

Proof. In the notation of Lemma 2.3 we have $|L|=|M(G)|\left|G^{\prime} \cap K\right|$, $e(L)$ divides $e(M(G)) e\left(G^{\prime} \cap K\right)$ and $d(L) \leqq d(M(G))+d\left(G^{\prime} \cap K\right)$.

The results now follow by Lemma 2.3(iii).

Corollary 3.2. Let $G$ be a finite d-generator group of order $p^{n}$. Then

$$
p^{d(d-1) / 2} \leqq|M(G)|\left|G^{\prime}\right| \leqq p^{n(n-1) / 2} .
$$

Proof. Suppose we take $K=\Phi(G)$, the Frattini subgroup of $G$, in part (i) of Theorem 3.1. Then $H$ is elementary abelian of order $p^{d}$ so that 
$|M(H)|=p^{d(d-1) / 2}$ (see, for example, [3]) and the left-hand inequality holds.

The right-hand inequality may be found in [4].

Corollary 3.2 gives some measure of the spread of $|M(G)|\left|G^{\prime}\right|$. In particular, it shows that the smaller the multiplicator of $G$ the larger the derived group of $G$, and conversely.

Suppose now that $G$ is a 3-generator $p$-group with trivial multiplicator. Then Corollary 3.2 shows that the order of $G^{\prime}$ is at least as large as $p^{3}$ so that $|G| \geqq p^{6}$. This means that 3-generator $p$-groups of orders no more than $p^{5}$ have nontrivial multiplicators. This fact can, of course, be obtained from tables of groups of orders dividing $p^{5}$.

It is often useful to know when a group has nontrivial multiplicator. Theorem 3.1 gives some sufficient conditions for a group to have nontrivial multiplicator and perhaps the most interesting is:

COROLlaRY 3.3. Let $G$ be a 3-generator finite p-group with $d\left(G^{\prime}\right)$ no more than 2. Then $M(G)$ is nontrivial.

Proof. If we take $K=\Phi(G)$ in Theorem 3.1(iii) then $H$ is elementary abelian of order $p^{3}$ so that

$$
d(M(H))=3 \leqq d(M(G))+d\left(G^{\prime}\right) \leqq d(M(G))+2 .
$$

The results now follows.

It is well known (see for example [3, p. 642]) that p-groups needing at least four generators have nontrivial multiplicators so that Corollary 3.3 extends the list of $p$-groups with nontrivial multiplicators.

Again, it follows immediately from Corollary 3.3 that 3-generator $p$-groups of order no more than $p^{5}$ have nontrivial multiplicators.

COROLlaRY 3.4. Let $G$ be a t-generator finite p-group. Then in any presentation for $G$ with $t$ generators and $\rho$ relations, we must have

$$
\rho \geqq \frac{1}{2} t(t+1)-d\left(G^{\prime}\right) .
$$

Proof. If a finite group $X$ can be presented with $n$ generators and $m$ relations, then it is well known (see [3, p. 642]) that $d(M(X)) \leqq m-n$.

Suppose then that $\rho<\frac{1}{2} t(t+1)-d\left(G^{\prime}\right)$ for some finite $t$-generator $p$ group $G$. Then

$$
\begin{aligned}
d(M(G)) & \leqq \rho-t<\frac{1}{2} t(t+1)-d\left(G^{\prime}\right)-t \\
& =\frac{1}{2} t(t-1)-d\left(G^{\prime}\right) .
\end{aligned}
$$

On the other hand, Theorem 3.1(iii), with $K=\Phi(G)$, shows that

$$
d(M(G)) \geqq \frac{1}{2} t(t-1)-d\left(G^{\prime}\right)
$$

so that we have a contradiction. 
4. Upper bounds. In this section, some upper bounds are given in terms of normal subgroups and factor-groups.

TheOREM 4.1. Let $G$ be a finite group and $B$ a central subgroup. Set $A=G / B$. Then

(i) $|M(G)|\left|G^{\prime} \cap B\right|$ divides $|M(A)||M(B)||A \otimes B|$,

(ii) $d(M(G)) \leqq d(M(A))+d(M(B))+d(A \otimes B)$,

(iii) $e(M(G))$ divides $e(M(A)) e(M(B)) e(A \otimes B)$.

Before proving Theorem 4.1, we make the following:

Definition 4.2. Let $X$ be a finite group. We say that $X$ has (special) rank $r(X)$ if every subgroup of $X$ may be generated by $r(X)$ elements and there is at least one subgroup that cannot be generated by fewer than $r(X)$ elements.

Proof OF THEOREM 4.1. Let $G=F / R$ be a presentation for $G$ as $a$ factor-group of the free group $F$ and let $B=S / R$ so that $A \cong F / S$. Then $[F, S] \leqq R$.

(i) By Lemma 2.3,

$$
|M(G)|\left|G^{\prime} \cap B\right|=|L|=|M(A)||[F, S] /[F, R]| .
$$

Now, $([F, S] /[F, R]) /\left([F, R] S^{\prime} /[F, R]\right)$ is isomorphic with $[F, S] /[F, R] S^{\prime}$. Hence,

But

$$
|M(G)|\left|G^{\prime} \cap B\right|=|M(A)|\left|[F, R] S^{\prime} /[F, R]\right|\left|[F, S] /[F, R] S^{\prime}\right| .
$$

$$
\begin{aligned}
{[F, R] S^{\prime} /[F, R] } & \cong S^{\prime} /\left(S^{\prime} \cap[F, R]\right) \\
& \cong\left(S^{\prime} /[S, R]\right) /\left(\left(S^{\prime} \cap[F, R]\right) /[S, R]\right)
\end{aligned}
$$

and, since $S^{\prime} \leqq[F, S] \leqq R$,

$$
S^{\prime} /[S, R]=\left(S^{\prime} \cap R\right) /[S, R] \cong M(B) .
$$

Hence (i) follows by Corollary 2.2 .

(ii) By Lemma 2.3 again

$$
\begin{aligned}
d(M(G)) \leqq r(L) & \leqq r(M(A))+r([F, S] /[F, R]) \\
& =d(M(A))+d([F, S] /[F, R]),
\end{aligned}
$$

since $M(A)$ and $[F, S] /[F, R]$ are finite abelian groups (for $[F, S]^{\prime} \leqq$ $\left.R^{\prime} \leqq[F, R]\right)$. The result now follows as for (i).

(iii) This again follows as for (i).

Note that if $B$ is cyclic, Theorem 4.1(i) generalises a result of J. A. Green [2] obtained by using the Lyndon spectral sequence.

COROLlaRY 4.3. Let $G$ be a finite $t$-generator group of order $p^{n}$. Then there exists an integer $h=h(G)$ with $t-1 \leqq h \leqq n-1$ such that

$$
d(M(G)) \leqq h t-\frac{1}{2} t(t-1) .
$$


Proof. If $G$ is cyclic, we obtain the result by choosing $h=0$. Suppose then that $G$ is noncyclic and choose $B_{1}$ in $Z(G)$ to be cyclic of order $e(Z(G))$. Then by Theorem 4.1(ii),

$$
d(M(G)) \leqq d\left(M\left(G / B_{1}\right)\right)+d\left(\left(G / B_{1}\right) \otimes B_{1}\right)=d\left(M\left(G / B_{1}\right)\right)+d\left(G / B_{1}\right) .
$$

For $j \geqq 2$, if $G / B_{j-1}$ is noncyclic, choose $B_{j} / B_{j-1}$ in $Z\left(G / B_{j-1}\right)$ to be cyclic of order $e\left(Z\left(G / B_{j-1}\right)\right)$.

Suppose $G / B_{k}$ is noncyclic for all $k$. By finiteness, there exists an integer $l$ such that $B_{l}=G$. Hence, $G / B_{l-1}=B_{l} / B_{l-1}$ and we have a contradiction.

Hence if $h$ is the minimum number of steps required to obtain cyclicity we have

$$
d(M(G)) \leqq \sum_{j=1}^{h} d\left(G / B_{j}\right)
$$

Since $e\left(Z\left(G / B_{j}\right)\right) \geqq p$ for all $j \geqq 0$ (where $B_{0}=1$ ), it is clear that $h \leqq n-1$. Also, $t \leqq h+1$ so that

$$
d(M(G)) \leqq \frac{1}{2} t(t-1)+\sum_{j=1}^{h-t+1} d\left(G / B_{j}\right) \leqq \frac{1}{2} t(t-1)+(h-t+1) t
$$

If $G$ is the direct product of $t$ finite cyclic $p$-groups then it is clear that $h=t-1$ so that the bound of Corollary 4.3 is attained by all finite abelian p-groups.

Corollary 4.3 generalises a result implicit in [6], but the two results coincide when $G$ is an elementary abelian $p$-group.

Let $G=F / R$ be a presentation for the finite $p$-group $G$ as a factorgroup of the free group $F$. Let $\Gamma_{j+1}=\gamma_{j+1}(F)$ for all $j$. Since $G^{\prime}=F^{\prime} R / R$ we have, by 1.1 , that

$$
M\left(G / G^{\prime}\right) \cong\left(F^{\prime} \cap F^{\prime} R\right) /\left[F, F^{\prime} R\right]=F^{\prime} /\left[F, F^{\prime} R\right] .
$$

With this notation we have:

THEOREM 4.4. Let $G$ be a finite p-group of nilpotency class $c$ and let $Q_{j}$ denote the quotient group $G / \gamma_{j}(G)$ for $2 \leqq j \leqq c$. Then

$$
\begin{aligned}
\left|G^{\prime}\right||M(G)| & \leqq\left|M\left(G / G^{\prime}\right)\right| \prod_{j=1}^{c-1}\left|Q_{j+1} \otimes \gamma_{j+1}(G)\right|, \\
d(M(G)) & \leqq d\left(M\left(G / G^{\prime}\right)\right)+\sum_{j=1}^{c-1} d\left(Q_{j+1} \otimes \gamma_{j+1}(G)\right), \\
e(M(G)) & \leqq e\left(M\left(G / G^{\prime}\right)\right) \prod_{j=1}^{c-1} e\left(Q_{j+1} \otimes \gamma_{j+1}(G)\right) .
\end{aligned}
$$


Proof. (i) In the above notation,

$$
\begin{aligned}
\left|G^{\prime}\right||M(G)| & =\left|F^{\prime}\right|[F, R]|=| M\left(G / G^{\prime}\right)||\left[F, F^{\prime} R\right] /[F, R] \mid \\
& =\left|M\left(G / G^{\prime}\right)\right|\left|\left[F, \Gamma_{j+2} R\right] /[F, R]\right| \prod_{k=1}^{j}\left|\left[F, \Gamma_{k+1} R\right] /\left[F, \Gamma_{k+2} R\right]\right|
\end{aligned}
$$

for all $j \geqq 1$. Now, $1=\gamma_{c+1}(G)=\Gamma_{c+1} R / R$ so that $\Gamma_{c+1} \leqq R$ and $\left[F, \Gamma_{c+1} R\right]=$ $[F, R]$. Next, $\gamma_{j}(G)=\Gamma_{j} R / R$ for all $j \geqq 2$. Thus

$$
[F, R]\left(\Gamma_{j} R\right)^{\prime}\left[F, \Gamma_{j} R, F\right]=[F, R] \Gamma_{j+2}=\left[F, \Gamma_{j+1} R\right]
$$

and (i) follows by Lemma 2.1 .

(ii) We have,

so that

$$
r\left(F^{\prime} /[F, R]\right) \leqq r\left(M\left(G / G^{\prime}\right)\right)+r\left(\left[F, \Gamma_{2} R\right] /[F, R]\right)
$$

$$
d(M(G)) \leqq d\left(M\left(G / G^{\prime}\right)\right)+\sum_{j=1}^{c-1} r\left(\left[F, \Gamma_{j+1} R\right] /\left[F, \Gamma_{j+2} R\right]\right),
$$

and (ii) again follows by Lemma 2.1 .

(iii) This follows as for (i) and (ii).

Note that part (i) of Theorem 4.4 gives a different result to that of [1], as can be seen by considering the groups $G(\alpha, \beta, \gamma), \gamma \geqq \alpha>\beta$, defined by $G(\alpha, \beta, \gamma)=\langle a, b, c, d, e, f\rangle$ with defining relations:

$$
\begin{aligned}
a^{p^{\gamma}} & =b^{p^{\alpha}}=c^{p^{\beta}}=d^{p^{\alpha}}=e^{p^{\beta}}=f^{p^{\beta}}=1 ; \\
a^{d} & =a, a^{e}=a, a^{f}=a, d^{e}=d, d^{f}=d, e^{f}=e ; \\
a^{b} & =a d, d^{b}=d, e^{b}=e, f^{b}=f ; \\
a^{c} & =a e, b^{c}=b f, d^{c}=d, e^{c}=e, f^{c}=f .
\end{aligned}
$$

Part (i) of Theorem 4.4 gives $|M(G)| \leqq p^{2(\alpha+2 \beta)+3 \beta}$ and [1] gives

where $G=G(\alpha, \beta, \gamma)$.

$$
|M(G)| \leqq p^{3(\alpha+2 \beta)},
$$

COROLlaRY 4.5. If $G$ is a finite p-group of special rank $r$ and nilpotency class $c$ then

$$
d(M(G)) \leqq \frac{1}{2} r((2 c-1) r-1) .
$$

Proof. If $d(G)=t$ then it follows from Theorem 4.4(ii) that

$$
d(M(G)) \leqq \frac{1}{2} t(t-1)+t \sum_{j=1}^{c-1} d\left(\gamma_{j+1}(G)\right) .
$$

The result now follows.

Note that the bound of Corollary 4.5 is attained by all abelian $p$-groups. As a final application of Theorem 4.4 we have: 
COROLlaRY 4.6. Further to the notation and assumptions of Theorem 4.4 let $e_{j}=\min \left\{e\left(Q_{j+1}\right), e\left(\gamma_{j+1}(G)\right)\right\}$ for $1 \leqq j \leqq c-1$. Then $e(M(G)) \leqq$ $e\left(G / G^{\prime}\right) \prod_{j=1}^{c-1} e_{j} ;$ in particular, if $G$ has exponent $p^{e}$ then $e(M(G)) \leqq p^{e c}$.

Proof. If $A$ is a finite abelian $p$-group then it is easy to see that $e(M(A))$ is no more than $e(A)$. Also, from the properties of tensor products (see [3]), it follows that $e\left(Q_{j+1} \otimes \gamma_{j+1}(G)\right) \leqq e_{j}$. The result follows.

I would like to thank the Science Research Council for its financial assistance and the referee for his useful comments.

\section{REFERENCES}

1. W. Gaschütz, J. Neubüser and Ti Yen, Über den Multiplikator von p-Gruppen, Math. Z. 100 (1967), 93-96. MR 36 \#272.

2. J. A. Green, On the number of automorphisms of a finite group, Proc. Roy. Soc. London Ser. A 237 (1956), 574-581. MR 18, 464.

3. B. Huppert, Endliche Gruppen I, Die Grundlehren der math. Wissenschaften, Band 134, Springer-Verlag, Berlin and New York, 1967. MR 37 \#302.

4. Michael R. Jones, Multiplicators of p-groups, Math. Z. 127 (1972), 165-166.

5. Roger C. Lyndon, The cohomology of group extensions, Duke Math. J. 15 (1948), 271-292. MR 10, 10.

6. P. M. Neumann, An enumeration theorem for finite groups, Quart. J. Math. Oxford Ser. (2) 20 (1969), 395-401. MR 40 \#7344.

7. I. Schur, Untersuchungen über die Darstellungen der endlichen Gruppen durch gebrochene lineare Substitutionen, J. Math. 132 (1907), 85-137.

Department of Mathematics, University College, Cardiff, South Wales, UNITED KINGDOM 